\title{
Modified Key Using Multi-Cycle Key In Vigenere Cipher
}

\author{
Ansar Rizal, Didi Susilo Budi Utomo, Rihartanto Rihartanto, Marselina Endah Hiswati, \\ Haviluddin Haviluddin
}

\begin{abstract}
Encryption-Decryption is one form of securing text data, images and sound in order to minimize data stealing, attack, etc. The purpose of this study is to modified encryption-decryption keys of Vigenere cipher.Based on experiment show that multi-cycle keyencryption-decryption have been implemented. Meanwhile, the best MAE values of final encryption process of 56.837 and final decryption process of 0 . In other words, the modification of key is more efficient and effective for data security. Furthermore, the multi-cycle key encryption-decryption optimizing using metaheuristics as one of the future works that can be conducted in order to investigate the best encryption-decryption especially using Vigenere cipher.
\end{abstract}

Keywords: Vigenere; encryption; decryption; multi-cycle; metaheuristics.

\section{INTRODUCTION}

Data security techniques are developed in order to minimize data stealing. Encryption is one form of securing text data, images and sound [1]. Then, data encryption is a process of changing the data into unreadable. So, the result of data encryption is cipher text. Meanwhile, in order to obtain information called decryption. Hence, a key is a main factor in encryption. In other words, the key is a heart of data security for decryption and encryption processes. Furthermore, an algorithm in order to process of encryption and decryption are divided into two types, namely symmetric and asymmetric keys. Symmetric is encryption and decryption process algorithm using the same key "one-key". Meanwhile, asymmetric key is an algorithm that uses two keys "different keys": private and public keys. The public key is the key for encryption. Then, private key is the key to decrypt cipher text. There are several types of symmetric algorithms including cipher (permutation and Substitution), cipher Hill, Caesar, Vigenere, OTP, RC6, Twofish, Magenta, FEAL, SAFER, LOCI, CAST, Rijndael (AES), Blowfish, GOST, A5, Kasumi, DES and IDEA, etc. [2]-[7].

Many studies using symmetric algorithm have been implemented. In order to improve its encryption and

decryption performances especially in an improvement

Revised Version Manuscript Received on 10 September, 2019. of Samarinda, Indonesia

Didi Susilo Budi Utomo, Department of Information Technology, State Polytechnic of Samarinda, Indonesia

Rihartanto Rihartanto, Department of Information Technology, State Polytechnic of Samarinda, Indonesia (E-Mail: rihart.c@gmail.com)

Marselina Endah Hiswati, Faculty of Science and Technology, Respati University of Yogyakarta, Indonesia

Haviluddin Haviluddin, Faculty of Computer and Information Technology, Mulawarman University, Indonesia
Ansar Rizal, Department of Information Technology, State Polytechnic

standard process of symmetric algorithm have also been carried out. For example, in Vigenere algorithm, by adding random bits (diffusion) before encryption on each byte [8], extend the Vigenere matrix [9], encryption repetitions [10], adding value to the plaintext[4], combining the LFSR key with Vigenere cipher key [6], using random table [7], varying the key [2] and more. Some researchers, such as [11] have been implemented Genetic Algorithm (GA), Particle Swarm Optimization (PSO) and Cuckoo Search (CS) algorithm as a crypt-analysis of Vigenere cipher using 26 characters. The results showed that GA and PSO techniques have recovered the entire key correctly for keys with small lengths. Then, CS technique has recovered more than $90 \%$ of key. In other words, CS showed that fast convergence and accuracy over PSO and GA.

In this study, Vigenere cipher as a development of the Caesar cipher algorithm especially on the filtering process will be applied. Meanwhile, the principle of Vigenere cipher algorithm is utilizing the Vigenere square for encryption. Classically, Vigenere cipher uses only 26 capital letters which starts from A to $\mathrm{Z}$ for encryption and decryption using "one-key". In other words, all characters like punctuations and numbers are unreadable. If the key has that characters (i.e., punctuations and numbers) so that it must be converted into capital letters. This is a weakness of Vigenere cipher algorithm which only use capital letters A-Z [5], [8], [9].

The aim of this study is to adjust the encryption-decryption keys in order to reduce the risk of weakness of Vigenere cipher algorithm using 128 ASCII characters. This paper is organized as follows. Research methodology is summarized in Section 2. Experimental results and discussions are given in Section 3, and Section 4 draws conclusions.

\section{MATERIAL AND METHODS}

\subsection{Vigenere Cipher}

In principle, a cryptographic algorithms can be classified into two types: classical and modern cryptographic [12]. Meanwhile, a Vigenere cipher method is a part of simple classical cryptographic. The Vigenere cipher is named after Blaise de Vigenere, a $16^{\text {th }}$ century Frenchman Diplomat. Later, first described by Giovan Batista Belaso in 1553. The Vigenere cipher is a polyalphabetic cipher (letter transformed to different letters), depending upon its position in the plaintext, when encoded [3], [13]. 


\subsection{ASCII Character}

ASCII characters stands for American Standard Codes for International Interchange which is a collection of codes that used to interaction between user and computer. ASCII is universal international standard code (i.e., codes, letters, and symbols) with 0-127 range values which have 7 bits of binary composition. In this study, the implementation of Vigenere cipher algorithm using 128 ASCII Character. It aims to prevent the loss of some data on plaintext such as null, beep, Del, backspace, enter and others because of the filtering process. The ASCII characters can be seen in Table 1. Where, 0-31 and 127 are control characters, and 32-126 are printable characters.

Table 1. ASCII Table

\begin{tabular}{|c|c|c|c|c|c|c|c|c|c|c|c|c|c|c|c|c|c|}
\hline Dec Hex & Oet & Chr & Dec & Hex & Oot & HTML & $\mathrm{Chr}$ & Dec & Hex & Oet & HTML & $\mathrm{Chr}$ & Deo & Hex & Oct & HTML & Chr \\
\hline 00 & 000 & NUII & 32 & 200 & 040 & 8प्र032; & Space & 64 & 40 & 100 & \&त्म064: & क & & 50 & 140 & 8प035: & \\
\hline 11 & 001 & Start of Header & & 21 & 041 & \& 2033 : & I & & 41 & 101 & 8Q065; & A & & 61 & 141 & 8N097: & a \\
\hline 22 & 002 & Start of Text & 34 & 22 & 042 & $8=034 ;$ & " & 66 & 42 & 102 & 8a066; & B & 98 & 62 & 142 & $8 \pm 098:$ & b \\
\hline 33 & 003 & End of Text & 35 & 23 & 043 & 84035: & " & 67 & & 103 & 8.0067: & c & 99 & 63 & 143 & 80099 & c \\
\hline 44 & 004 & End of Transmission & 36 & & 044 & 20036 & s & 68 & & 104 & \&\$068: & D & 100 & 64 & 144 & $8 \geqslant 100$. & d \\
\hline 55 & 005 & Enquiry & 37 & 25 & 045 & \&4037: & $\%$ & 69 & 45 & 105 & 8xa069: & E & 101 & 65 & 145 & 8.0101: & e \\
\hline 66 & 006 & Acknowledgment & 38 & 26 & 046 & $8=038$ & $\&$ & 70 & 46 & 106 & 80070 & $\mathbf{F}$ & 102 & 66 & 146 & $8 \pm 102$ & $f$ \\
\hline 77 & 007 & Bell & 39 & 27 & 047 & \& & & 71 & 47 & 107 & \& $+071:$ & G & 103 & 67 & 147 & $8 \# 103$ & 9 \\
\hline 88 & 010 & Backspace & 40 & 28 & 050 & $8+0=0$ & s & 72 & 48 & 210 & 8.072: & H & 104 & 68 & 150 & 8*104: & $h_{h}$ \\
\hline 99 & 011 & Horizontal Tab & 41 & 29 & OS1 & \&®041: & j & 73 & 49 & 111 & 80073: & I & 105 & 69 & 151 & \&\#105: & 1 \\
\hline $10 \mathrm{~A}$ & 012 & Line feed & 42 & $2 \mathrm{~A}$ & 052 & $2 \neq 042$ & * & & $4 \mathrm{~A}$ & 112 & $8 \times 074$ & J & 106 & $6 \mathrm{~A}$ & 152 & $8+106$ & j. \\
\hline $11 \mathrm{~B}$ & 013 & Vertical Tab & 43 & $2 B$ & 053 & Q $=043$ & + & 75 & 48 & 113 & 8.075: & K & 107 & $6 B$ & 153 & 2)107. & k \\
\hline $12 \mathrm{C}$ & 014 & Form feed & 44 & $2 C$ & 054 & $8 \times 044:$ & 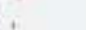 & 76 & $4 \mathrm{C}$ & 114 & 89076 & $i$ & 108 & $6 C$ & 154 & SN108 & $i$ \\
\hline 130 & 015 & Carriage return & 45 & 20 & 055 & $2 \neq 045$ & - & & 4D & 115 & 8.077: & M & 109 & 60 & 155 & $8 * 109$. & m \\
\hline $14 \mathrm{E}$ & 016 & Shift Out & 46 & $2 E$ & 056 & 8N046: & & 78 & $4 E$ & 116 & 86078 & $\mathrm{~N}$ & 110 & $6 \mathrm{E}$ & 156 & 80110 & n \\
\hline $15 \bar{F}$ & 017 & Shift In & 47 & $2 F$ & 057 & 8.047: & 1 & 79 & $4 \mathrm{~F}$ & 117 & \& 1079 : & 0 & 111 & $6 F$ & 157 & 84111: & 0 \\
\hline 1610 & 020 & Data Link Escape & 48 & 30 & 060 & 8. 048 & 0 & 80 & 50 & 120 & 8xt080: & $\vec{p}$ & 112 & 70 & 160 & $8+112$ & p \\
\hline 1711 & 021 & Device Control 1 & 49 & 31 & 061 & 84049: & 1 & 81 & & 121 & Q⿻日乚: & $Q$ & 113 & 71 & 161 & \&N113: & $\mathrm{q}$ \\
\hline 1812 & 022 & Device Control 2 & 50 & 32 & 062 & Q & 2 & 82 & 52 & 122 & $8=082$ & R & 114 & 72 & 162 & $8 \geqslant 114$ & $r$ \\
\hline 1913 & 023 & Device Control 3 & 51 & 33 & 063 & \&e051: & 3 & 83 & 53 & 123 & $8 \times 083:$ & s & 115 & 73 & 163 & 8.115: & s \\
\hline 2014 & 024 & Device Control 4 & 52 & & 064 & $8=052:$ & 4 & 84 & 54 & 124 & \& $2+084:$ & $T$ & 116 & 74 & 164 & $8 \geqslant 116$ & $\hat{t}$ \\
\hline 2115 & 025 & Negative Ack. & 53 & 35 & 065 & Quos 3 ; & 5 & 85 & 55 & 125 & 2ar085: & $\mathbf{u}$ & 117 & 75 & 165 & $\operatorname{sen} 117$ & u \\
\hline 2216 & 026 & Synchronous idle & 54 & 36 & 066 & $8=054$ & 6 & 86 & & 126 & $\&=086$ & v & 118 & 76 & 166 & $8 \times 118$ & v \\
\hline 2317 & 027 & End of Trans. Block & s5 & 37 & 067 & 8no5s: & 7 & 87 & 57 & 127 & 84087: & $w$ & 119 & 77 & 167 & 8)119. & w \\
\hline 2418 & 030 & Cancel & 56 & 38 & 070 & 8.056: & 8 & 88 & 58 & 130 & 8.2088: & $\mathrm{x}$ & 120 & 78 & 170 & $8=120$ & $x$ \\
\hline & 031 & End of Medium & 57 & 39 & 071 & $8=057$ & 9 & 89 & & 131 & \&.089: & $\mathrm{\gamma}$ & 121 & 79 & 171 & \&\#121: & $y$ \\
\hline $261 \mathrm{~A}$ & 032 & Substitute & 58 & $3 A$ & 072 & 8,058 & $i$ & 90 & $5 A$ & 132 & 80090 & $z$ & 122 & $7 \mathrm{~A}$ & 172 & $8 \times 122$ & $z$ \\
\hline 2718 & 033 & Escape & 59 & $3 B$ & 073 & $8 \times 059$ & 2 & 91 & $5 B$ & 133 & $8=091$ & t & 123 & 78 & 173 & 8थ123; & i \\
\hline $281 C$ & 034 & File Separato & 60 & $3 \mathrm{C}$ & 074 & 86060: & $<$ & 92 & & 134 & \&o092; & i & 124 & $7 C$ & 174 & $8 \times 124$ & \\
\hline 2910 & 035 & Group Separator & 61 & 30 & 075 & Q4061: & $=$ & 93 & 5D & 135 & Q 20093 & $i$ & 125 & 70 & 175 & $8 * 125$ & $\mathrm{i}$ \\
\hline $30 \mathrm{IE}$ & 036 & Record Separator & 62 & $3 E$ & 076 & Q $2=062$ & $>$ & 94 & SE & 136 & 8c0094; & A & 126 & $7 E$ & 176 & 8n126: & - \\
\hline 31 if & 037 & Unit Separator & 63 & $3 F$ & 077 & \& & 7 & 95 & $5 F$ & 137 & 8.0095; & & 127 & $7 F$ & 177 & 8. 127 . & Del \\
\hline
\end{tabular}

Source: http://www.asciichars.com

In this paper, short key modifications are used in order to be retained in the encrypted text. In this study, the encryption process using Eq. 3, and the decryption process using Eq. 4 are presented. These formulas are Vigenere cipher classical algorithm modifications, Eq. 1, 2.

$$
\begin{aligned}
C_{i} & =\left(P_{i}+K_{i}\right)-26 \\
P_{i} & =\left(C_{i}-K_{i}\right)+26 \\
C_{i} & =\left(P_{i}+K_{i}\right) \bmod 128 \\
P_{i} & =\left(C_{i}-K_{i}\right) \bmod 128
\end{aligned}
$$

As an illustrated. The original plaintextdata name is applied "Polytechnic" with key of "ab1d". Firstly, plaintext

\begin{tabular}{|c|c|c|c|c|c|c|c|c|c|c|c|}
\hline \multirow[t]{2}{*}{ C'pheterten (C) } & 1 & l & & 1 & $\pi$ & G & & L & 0 & $\mathrm{~K}$ & \\
\hline & 49 & 81 & 29 & 93 & 85 & 71 & $x$ & 76 & 79 & 75 & 20 \\
\hline \multirow[t]{2}{*}{$\operatorname{Reg}(\mathbb{K})$} & 2 & $b$ & 1 & $t$ & $i$ & $b$ & 1 & $d$ & a & $b$ & 1 \\
\hline & 97 & 98 & 49 & 100 & 97 & 98 & 49 & 102 & 97 & 98 & 49 \\
\hline C-Kmod 128 & 81 & 111 & 108 & 121 & 116 & 101 & 99 & 104 & 110 & 105 & 99 \\
\hline Plintert [I] & P & 0 & 1 & $y$ & t & e & c & b & II & i & i \\
\hline
\end{tabular}
and key is changed into ASCII character using Eq. 3 and 4. The encryption-decryption processes of Vigenere cipher as an illustrated bellow.

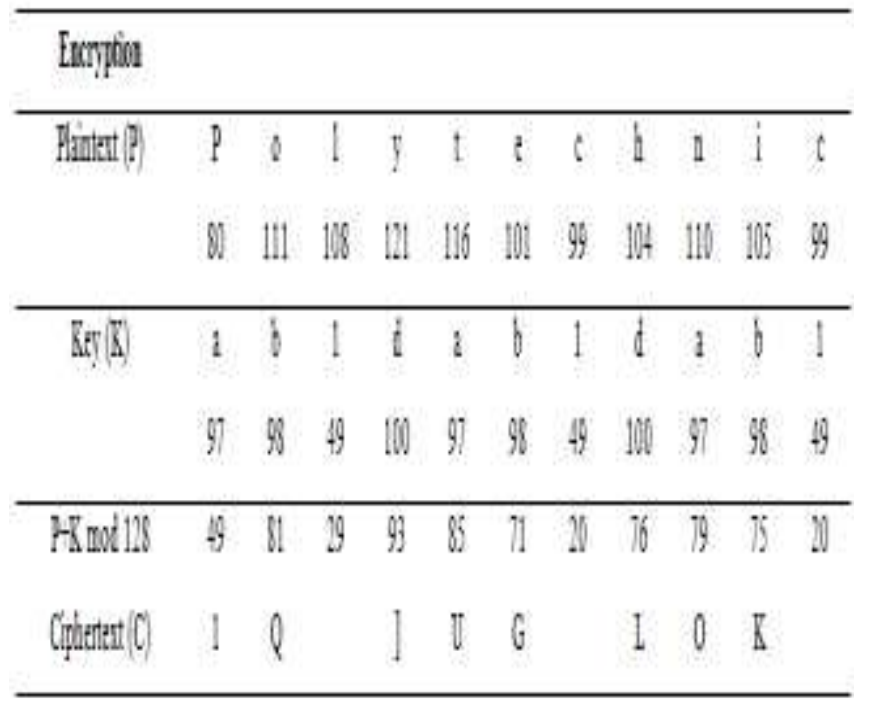

\subsection{Multi Cycle Encryption and Decryption Processes}

In this study, multi-cycle encryption has been implemented. The goal is to improve the performance of Vigenere cipher without additional algorithm. Where, the keys for multi-cycle process have been created from the initial key using permutation. The flow of encryption-decryption multi-cycle process can be seen in Figure 1. 

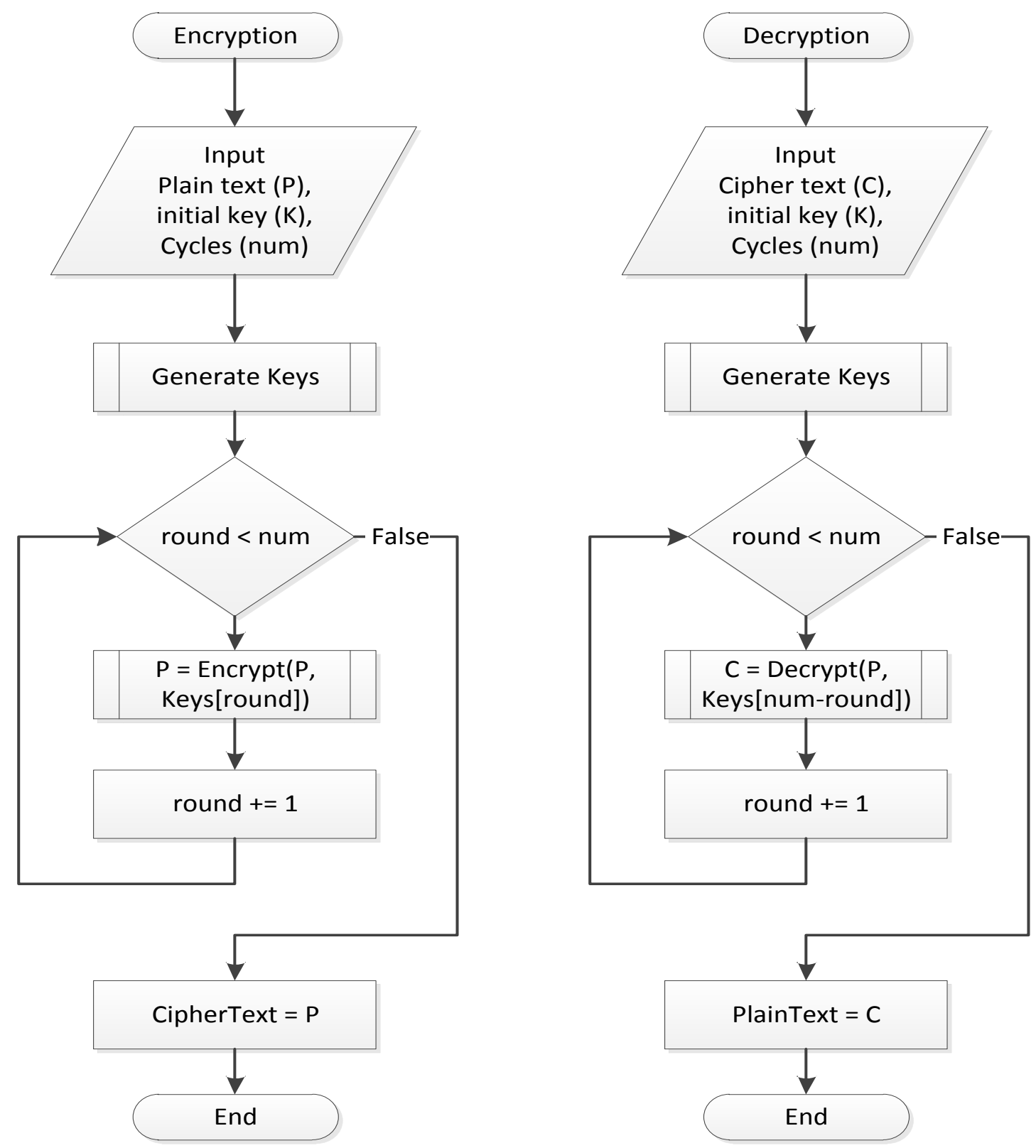

Figure 1. Flowchart of encryption-decryption multi-cycle in Vigenere cipher

In the encryption process, the ciphertext as output from the first cycle will become the plaintext for the second cycle, and so on until the last cycle. The same manner is applied to the decryption process.

\subsection{Performance Measurement}

In this study, the performance of Vigenere cipher algorithm is measured using Mean Absolute Error (MAE). Where, the MAE value is obtained from the difference original character and substitution distance positions. In this case, it is plaintext to ciphertext changed. The MAE formula using Eq. 5.

$$
M A E=\frac{\sum_{i=1}^{n}\left|y_{i}-x_{i}\right|}{n}
$$

Where, $\mathrm{n}$ is the number of characters; $\mathrm{y}_{\mathrm{i}}$ is the cipher character; $x_{i}$ is the plain character.

\section{RESULTS}

In this experiment, the encryption-decryption program created using Pythonprogramming in order to run and analyze the Vigenere cipher algorithm was explored.Theitertools module of python language for the permutation has been exploited. The keys from the initial key using permutation in order to produce unique keys which have the same length with the initial key then selected randomly have been generated. In order to ensure that the selected keys were exactly the same for the encryption and decryption process, a seed random was specified based on the given initial key. The seed random value was derived from the sum of the ASCII values multiplied by its position for each character contained in the initial key. It is assumed that the minimum initial key length was six characters 
consisting of at least three different characters, the goal some combination of keys that can be selected for use in the encryption process were obtained.
The tests on five different plaintext using the same initial key were carried out. The initial key was "abc123" and the plaintext as shown in Table 2.

Table 2. The Plaintexts

\begin{tabular}{|c|c|c|}
\hline Plaintext & \# Chars & Description \\
\hline Cryptography is about securing information. & 43 & Regular sentence \\
\hline abcdef 123456 abcdef 123456 abcdef 123456 & 41 & Two word segment,repeated for three times \\
\hline abc123abc123abc123abc123abc123abc123 & 36 & The initial key, repeated for six times \\
\hline def4567def4567def4567def4567def4567 & 35 & $\begin{array}{l}\text { "def } 4567 \text { " one character longer than the initial key, repeated for } \\
\text { five times }\end{array}$ \\
\hline greengreengreengreengreengreengreen & 35 & $\begin{array}{l}\text { "green" one character lesser than the initial key, repeated for } \\
\text { seven times }\end{array}$ \\
\hline $\begin{array}{l}\text { For the processes encryption-decrypti } \\
\text { cycles of Vigenere cipher encryption-de }\end{array}$ & & $\begin{array}{l}\text { presented. From the given initial key "abc123' then the } \\
\text { selected generated keys were "a123cb", " } 1 \mathrm{a} 2 \mathrm{c} 3 \mathrm{~b} \text { " and } \\
\text { " } 2 \mathrm{bc} 31 \mathrm{a} \text { " }\end{array}$ \\
\hline
\end{tabular}

Table 3. Result of Encryption

\begin{tabular}{|c|c|c|c|c|}
\hline Cycle & Key & Plaintext & Ciphertext & $\begin{array}{l}\text { MeanAbsolute } \\
\text { Error (MAE) }\end{array}$ \\
\hline
\end{tabular}

\section{The first plaintext}

$1 \quad$ a123cb Cryptography is about securing information.

$\begin{array}{lll}2 & 1 \mathrm{a} 2 \mathrm{c} 3 \mathrm{~b} & \begin{array}{l}\text { \$\#+\#WQH\#\#[\%SDDP\&\&SVGD\&\$QI } \\ \text { RTN\&RP }\end{array} \\ & & \mathrm{U}] \\ 3 & 2 \mathrm{bc} 31 \mathrm{a} & \begin{array}{l}3 \mathrm{yE} \sim=2\{\mathrm{~W} 6 \mathrm{w} \& \mathrm{X} 6) \mathrm{uV} \square+2\{\mathrm{R} \mid 6 \square \mathrm{XX} \square 2 @\end{array}\end{array}$

The second plaintext

$1 \quad$ a123cb abcdef 123456 abcdef 123456 abcdef 123456

$21 \mathrm{a} 2 \mathrm{c} 3 \mathrm{~b} \quad$ BHHbdfQFFFRdfhSDDDdfh 32 2bc31a $\begin{array}{ll}\text { stGz }\{* 2 \mathrm{CIJyH} 2 \mathrm{Exy}(\mathrm{wxGHwFG6w \& uvI} \mid 6 \mathrm{uDE} \\ \mathrm{KL}\end{array}$

\section{The third plaintext}

1 a123cb abc123abc123abc123abc123abc123abc123

$2 \quad 1 \mathrm{a} 2 \mathrm{c} 3 \mathrm{~b} \quad \mathrm{BdBdBdBdBdBd}$

3 2bc31a stGGHwstGGHwstGGHwstGGHwstGGHwst GGHw

\section{The fourth plaintext}

$1 \quad$ a123cb def4567def4567def4567def4567def4567

2 1a2c3b EghIgiHHfhjGGGegi

32 2bc31a vwJJKzIvI $\mid J y H I H\{\mid x G H z\{* F G M z) x F L M$

The fifth plaintext

1 a123cb greengreengreengreengreengreengreen

$21 \mathrm{a} 2 \mathrm{c} 3 \mathrm{~b}$ H\#QIS!JTF UGF\%HGO\$HPH\#Q

$32 \mathrm{bc} 31 \mathrm{a} \quad \mathrm{yI}\{+\mathrm{wI}\} 6 \mathrm{wwR}\}) \mathrm{wK}\{) \mathrm{yV}\{\{2 \mathrm{yI}\{$
\$\#+\#WQH\#\#K[\%SDDP\&\&SVG D\&\$QI RTN\&RP

52.256

U] $3 y \mathrm{E} \sim=2\{\mathrm{~W} 6 \mathrm{w} \& \mathrm{X} 6$

) $\mathrm{uV} \square+2\{\mathrm{R} \mid 6 \square \mathrm{XX} \square 2 @$

52.140

f@ $9 ;+\mathrm{f}(9 /-\mathrm{d}]: \mathrm{i}(3 \mathrm{i} ; \mathrm{i}:$

'i925d]5/61U;26r

56.837

BHHbdfQFFFRdfhSDDDdfh

46.390

stGz $\{$ *2CIJyH2Exy(wxGHwFG6

w\&uvI|6uDEKL

27.805

$\% \mathrm{~V}^{*}, \mathrm{~d} \% \mathrm{y} \mid\left\{\mathrm{Zz}\left(+^{*}\right)\right.$

ZgzyXx) $\} \mathrm{i}\left({ }^{\prime} \mathrm{X}, / \mathrm{gVv}\{\sim\}\right.$

stGGHwstGGHwstGGHwstGGH wstGGHwstGGHw

$\% \mathrm{~V} *_{\mathrm{zy}} \%_{\mathrm{V}} *_{\mathrm{zy}} \% \mathrm{~V} *_{\mathrm{zyX}} \% \mathrm{~V} *_{\mathrm{z}}$ $\mathrm{yX} \% \mathrm{~V}^{*}{ }_{\mathrm{zyX}} \% \mathrm{~V}^{*} \mathrm{zyX}$

51.667

EghIgiHHfhjGGGegi

46.629

vwJJKzIvI |JyHIH $\{\mid x G H z\{$ *FGMz

)xFLM

27.771

$(\mathrm{Y}-\} \mid\left[\left\{\mathrm{X}, /\left\{\mathrm{Zz}++.-\mathrm{Yy}^{*} \sim-, \quad \mathrm{x}\right)\right\}+\right.$

$*(\mid \square \sim$

54.543

H\#QIS!JTF UGF\%HGO\$HPH\#Q

yI $\{+\mathrm{wI}\} 6 \mathrm{wwR}\}) \mathrm{wK}\{) \mathrm{yV}\{\{2 \mathrm{yI}\{$

54.314

)b.;

$2[9 .,+\mathrm{f}, .5$ 
Table 4. Result of Decryption

\begin{tabular}{|c|c|c|}
\hline Cycle & Key & Ciphertext \\
\hline \multicolumn{3}{|c|}{ The first plaintext } \\
\hline 1 & $2 \mathrm{bc} 31 \mathrm{a}$ & $\begin{array}{l}\text { f@ } 9 ;+f(9 /-d]: i(3 i ; i: \\
\text { 'i925d]5/61U;26r }\end{array}$ \\
\hline 2 & $1 a 2 c 3 b$ & $\begin{array}{l}\mathrm{U}] \\
3 \mathrm{yE} \sim=2\{\mathrm{~W} 6 \mathrm{w} \& \mathrm{X} 6) \mathrm{uV} \square+2\{\mathrm{R} \mid 6 \square \mathrm{x} \square Q @\end{array}$ \\
\hline 3 & a123cb & $\begin{array}{l}\text { \$\#+\#WQH\#\#K[\%SDDP\&\&SVGD\&\$QI } \\
\text { RTN\&RP }\end{array}$ \\
\hline
\end{tabular}

The second plaintext

$\begin{array}{lll}1 & \text { 2bc31a } & \% V^{*-}, \\ & & \text { d\%yl\{Zz(+*)ZgzyXx) }\} \text { i('X,/gVv' }\{\sim\} \\ 2 & \text { 1a2c3b } & \text { stGz }\{* 2 \text { CIJyH2Exy }(\text { wxGHwFG6w\&uvI } \mid 6 u D E \\ & & \text { KL } \\ 3 & \text { a123cb } & \text { BHHbdfQFFFRdfhSDDDdfh }\end{array}$

U] $\quad 3 y \mathrm{E} \sim=2\{\mathrm{~W} 6 \mathrm{w} \& \mathrm{X} 6$

) $\mathrm{uV} \square+2\{\mathrm{R} \mid 6 \square \mathrm{sX} \square 2 @$

\$\#+\#WQH\#\#K[\%SDDP\&\&SVG

D\&\$QI RTN\&RP

Cryptography is about securing information.

Mean Absolute
Error (MAE)

stGz\{*2CIJyH2Exy(wxGHwFG6

w\&uvI 6 UDEKL

52.140

52.256

0.000

BHHbdfQFFFRdfhSDDDdfh

27.805

abcdef 123456 abcdef 123456

abcdef 123456

0.000

The third plaintext

\begin{tabular}{|c|c|c|}
\hline & $2 \mathrm{bc} 31 \mathrm{a}$ & $\begin{array}{l}\% \mathrm{~V} * \mathrm{zy} X \% \mathrm{~V}^{*} \mathrm{zy} X \% \mathrm{~V} * \mathrm{zyX} \% \mathrm{~V} * \mathrm{zyX} \% \mathrm{~V} * \mathrm{zyX} \\
\% \mathrm{~V} *_{\mathrm{zy} X}\end{array}$ \\
\hline & $1 a 2 c 3 b$ & $\begin{array}{l}\text { stGGHwstGGHwstGGHwstGGHwstGGHwst } \\
\text { GGHw }\end{array}$ \\
\hline & $123 \mathrm{cb}$ & BdBdBdBdBdBd \\
\hline
\end{tabular}

stGGHwstGGHwstGGHwstGGH wstGGHwstGGHw

BdBdBdBdBdBd

49.667

abc123abc123abc123abc123abc12 $3 \mathrm{abc} 123$

0.000

\section{The fourth plaintext}

$\begin{array}{lll}1 & \text { 2bc31a } & (\mathrm{Y}-\} \mid\left[\left\{\mathrm{X}, /\left\{\mathrm{Zz}++.-\mathrm{Yy}^{*} \sim-, \mathrm{x}\right)\right\}+*(\mid \square \sim\right. \\ 2 & 1 \mathrm{a} 2 \mathrm{c} 3 \mathrm{~b} & \text { vwJJKzIvI } \mid \mathrm{JyHIH}\{\mid \mathrm{xGHz}\{* \mathrm{FGMz}) \mathrm{xFLM} \\ 3 & \text { a123cb } & \text { EghIgiHHfhjGGGegi }\end{array}$
vwJJKzIvI|JyHIH $\{\mid x G H z\{$ FGMz xFLM
EghIgiHHfhjGGGegi
def4567def4567def4567def4567d ef4567

0.000

\section{The fifth plaintext}

$\begin{array}{lll}1 & \text { 2bc31a } & +f, .56 Y, 7 .) Y 509) b . ;, 2[9 .,+f, .5 \\ 2 & 1 a 2 c 3 b & \text { yI }\{+w I\} 6 w w R\}) w K\{) y V\{\{2 y I\{ \\ 3 & \text { a123cb } & \text { H\#QIS!JTF UGF\%HGO\$HPH\#Q }\end{array}$

$\mathrm{yI}\{+\mathrm{wI}\} 6 \mathrm{wwR}\}) \mathrm{wK}\{) \mathrm{yV}\{\{2 \mathrm{yI}\{$

H\#QIS!JTF UGF\%HGO\$HPH\#Q

54.686

greengreengreengreengreengreeng reen

0.000

In this experiment the performance of encryption-decryption using mean absolute error (MAE) have been used.The MAE value of encryption process vary depend on the plaintext. These MAE values from its original plaintext were measured. All MAE values were greater than 0 , it mean that all cipher texts were different from its original. Then, the MAE value of final decryption of 0 , means that the text decryption was the same as the original.

In this experiment, characteristic checking for cryptanalysis has also been performed. Then, the character check results that there were no duplicated characters that have same as key lengths have been showed. Therefore, the original character was unpredictable especially using the Kasiski or Friedman Test methods. The results of character repeating can be seen in Table 5 for the first plaintext which is a regular sentence. Since there weretoo many repeating characters for the second to the fifth plaintext, thus the summary its occurrence, maximum and minimum character in each cycle is shown in Table 6. It is interesting, for example in the second and the fourth plaintext, while in the beginning there were so many repeating characters in the original plaintext, then extremely reduce in the first cycle and no repeating at all in the second of the third cycle. 
MODIFIED KEY USING MULTI-CYCLE KEY IN VIGENERE CIPHER

Table 5. Results of Characters Repeating Positionsfor the First Plaintext

\begin{tabular}{cccc}
\hline Text & Characters & Length & Occurrences \\
\hline Plaintext & "in" & 2 & 2 \\
& " i" & 2 & 2 \\
CipherText1 & "" & 2 & 2 \\
CipherText2 & "2\{" & 2 & 2 \\
CipherText3 & "d]" & 2 & 2 \\
\hline
\end{tabular}

Table 6. Number of Occurrence Characters in Each Cycle

\begin{tabular}{|c|c|c|c|c|c|}
\hline & & $2^{\text {nd }}$ plain text & $3^{\text {rd }}$ plain text & $4^{\text {th }}$ plain text & $5^{\text {th }}$ plain text \\
\hline \multirow[t]{3}{*}{ Original text } & \# of words & 182 & 87 & 91 & 70 \\
\hline & $\max$ chars & 14 & 18 & 14 & 15 \\
\hline & min chars & 2 & 2 & 2 & 2 \\
\hline \multicolumn{6}{|l|}{$1^{\mathrm{st}}$} \\
\hline \multirow{3}{*}{$\begin{array}{l}\text { Ciphertext } \\
\text { (first cycle) }\end{array}$} & \# of words & 4 & 87 & 2 & 10 \\
\hline & $\max$ chars & 2 & 18 & 2 & 5 \\
\hline & min chars & 2 & 2 & 2 & 2 \\
\hline \multicolumn{6}{|l|}{$2^{\text {nd }}$} \\
\hline \multirow{3}{*}{$\begin{array}{c}\text { Ciphertext } \\
\text { (second cycle) }\end{array}$} & \# of words & - & 87 & - & 10 \\
\hline & max chars & - & 18 & - & 5 \\
\hline & min chars & - & 2 & - & 2 \\
\hline \multicolumn{6}{|l|}{$3^{\mathrm{rd}}$} \\
\hline \multirow{3}{*}{$\begin{array}{l}\text { Ciphertext } \\
\text { (third cycle) }\end{array}$} & \# of words & - & 87 & - & 10 \\
\hline & max chars & - & 18 & - & 5 \\
\hline & min chars & - & 2 & - & 2 \\
\hline
\end{tabular}

\section{DISCUSSION}

In this experiment, the first plaintext using a regular expression have been used, while the other was a text constructed from a sequence of repetitive characters. In addition, for the same keys were used successively on each encryption cycle, and in descending order of each decryption cycle, Table 2.

Tables 3 and 4 show that every keys were used successively in each encryption cycle, and in reverse order in each decryption cycle. The first cycle encrypts the original plaintext. Then, in the second cycle was the first cycle output as plaintext has been used.

Then, the third cycle was the second cycle output as plaintext has been used, and so forth, while there were more cycles have been applied.

In contrast to [9] suggested that a one-time pad on a Vigenere cipher, where its length is as long as the key is true. Nevertheless, in this experiment, using short keys and which may require minimal cryptanalysis in each ciphertext of each cycle can be overcome by key modification.

\section{CONCLUSION}

This paper has presented the performance of classic Vigenerecipher implementing 128 ASCII characters with multi-cycle key encryption-decryption. The mean absolute error (MAE) are computed for each key encryption-decryption model and compared. Based on the results obtained, the multi-cycle key is found to be more efficient in encryption-decryption using Vigenere cipher algorithm. Optimizing the multi-cycle key encryption-decryption such as calculating of multi-cycles and key length as one of the future works that can be conducted in order to investigate the best encryption-decryption in Vigenere cipher

\section{REFERENCES}

1. A. Jawahir and H. Haviluddin, "An Audio Encryption Using Transposition Method," Int. J. Adv. Intell. Informatics, vol. 1, no. 2, July 2015, pp. 98-106, 2015.

2. Q.-A. Kester, "A cryptosystem based on Vigenère cipher with varying key," Int. J. Adv. Res. Comput. Eng. Technol., vol. 1, no. 10, pp. 108-113, 2012.

3. Q.-A. Kester, "A Hybrid Cryptosystem Based on Vigenère Cipher and Columnar Transposition Cipher," Int. J. Adv. Technol. Eng. Res., vol. 3, no. 1, pp. 141-147, 2013.

4. A. A. Soofi, I. Riaz, and U. Rasheed, "An Enhanced Vigenere Cipher For Data Security,” Int. J. Sci. Technol. Res., vol. 5, no. 3, pp. 141-145, 2016.

5. R. S. Kartha and V. Paul, "Survey: Recent Modifications in Vigenere Cipher," IOSR J. Comput. Eng., vol. 16, no. 2, pp. 49-53, 2014.

6. A. Razzaq, Y. Mahmood, F. Ahmed, and A. Hur, "Strong Key Machanism Generated by LFSR based Vigenère Cipher,” Int. Arab Conf. Inf. Technol., pp. 554-548, 2012.

7. Y. K. Singh, "Generalization of Vigenere cipher," ARPN J. Eng. Appl. Sci., vol. 7, no. 1, pp. 39-44, 2012.

8. P. Wilson and M. Garcia, "A Modified Version of the Vigenère Algorithm," Int. J. Comput. Sci. Netw. Secur., vol. 6, no. 3, pp. 140-143, 2006. 
9. P. Ravindra, B. Kallam, S. U. Kumar, A. Vinaya, and V. Shravan, "A Contemporary Polyalphabetic Cipher using Comprehensive Vigenere Table,” World Comput. Sci. Inf. Technol. J., vol. 1, no. 4, pp. 167-171, 2011.

10. R. S. Kartha and V. Paul, "A New Cryptosystem Based On Polyalphabetic Substitution Scheme With Multiple Number Of Cipher," in 6th IRF International Conference, 2014, pp. 40-44.

11. A. K. Bhateja, A. Bhateja, S. Chaudhury, and P. K. Saxena, "Cryptanalysis of Vigenere cipher using Cuckoo Search," Appl. Soft Comput. J., 2015.

12. H. Delfs, K. Paterson, and R. Cramer, Introduction to Cryptography: Principles and Application, Third Edit. Berlin: Springer-Verlag GmnH, 2015.

13. K. Senthil, K. Prasanthi, and R. Rajaram, "A modern avatar of Julius Ceasar and Vigenere cipher," 2013 IEEE Int. Conf. Comput. Intell. Comput. Res. IEEE ICCIC 2013, pp. 13-15, 2013. 